\title{
BROWSER'S NOTES
}

(C) ISS 2010

\section{Reliability of Clinical findings and magnetic resonance imaging for the diagnosis of chondromalacia patellae Pihlagamaki $H K$, et al. JBJS (Am) 2010:92:927-934}

56 young adults (median age 19.5 years) with anterior knee pain and MRI demonstrated chondral lesions were compared with arthroscopy. Arthroscopy confirmed chondromalacia in 25 patients $(45 \%)$. No association was found between seventy with chondromalacia seen at arthroscopy and symptoms of anterior knee pain. Sensitivity with a 1 OT MRI was low for grade I lesions but considerably higher for grades II to IV. The authors conclude that chondromalacia patellae cannot be diagnosed on the basis of current physical examination methods. Thus symptoms of anterior knee pain should not be an indication for knee arthroscopy. MRI may be considered an accurate diagnostic tool for identification of more severe cases of chondromalacia patellae.

Factors involved in the development of osteoarthritis after ACL surgery

Keays SL, et al. Am J. Sports Med (2010) 38: 455-463

Nearly $50 \%$ of patients develop mild to moderate OA after ACL repairs. Five factors were predictive of tibiofemoral OA; meniscectomy $(r=.72)$ and chondral damage $(r=41)$ were the strongest discriminators. This was closely followed by patellar tendon grafting. Weak quadriceps and low quadriceps to hamstring strength were very close discriminators. For patellofemoral OA, meniscectomy, chondral damage and age at surgery were the strongest predictors.

\section{Does the literature confirm superior clinical results in radiographically healed rotator cuffs after rotator cuff repair?}

Slabaugh MA, et al. Arthroscopy (2010) 26:393-403

Thirteen studies of arthroscopically repaired rotator cuffs were analysed (5 ultrasound, $4 \mathrm{MRI}$, and $2 \mathrm{CTA}$ and 2 combined CTA/MRI) to clarify the correlation between structural integrity (i.e. recurrent or persistent defects) and clinical outcomes. Open repairs, subscapularis repairs and studies that did not define outcomes based on healing or non-healing were excluded. Improvement was measured on strength, range of motion and post-operative shoulder scores, patient satisfaction. 1472 of 1931 repaired shoulders were evaluated post-operatively for the presence of a defect. $79.6 \%$ had an intact cuff. The authors conclude that there are some important differences in clinical outcomes between healed and non-healed repaired rotator cuffs given the variables in the studies analysed.

\section{Diagnosing suspected scaphoid fractures \\ Yin $Z G$, et al. \\ CORR (2010) 468:723-734}

There is marked inconsistency between institutions and amongst investigators in the choice of imaging for suspected scaphoid fractures. A meta-analysis of 26 studies was performed to compare diagnostic performances of scintigraphy, MRI and CT for diagnosing scaphoid fracture. Most studies had some methodologic limitations. Only two studies met $70 \%$ of the criteria (nine items) and five studies met fewer then $50 \%$ (seven items). Only English language reports were analysed. The limitations of the studies analysed were applying the commonly used standard of absence of a scaphoid fracture 6 weeks after injury and reporting of the reference test with knowledge of the test result. MRI and CT had high sensitivities and specificities. Bone scintigraphy had comparable sensitivity but lower specificity. Because the authors encountered imprecision in estimating CT sensitivity they concluded that MRI was the best test for confirming a scaphoid fracture with scintigraphy and MRI showing equal sensitivity for excluding a fracture.

Abstracted by M. Sundaram, M.D. October 2010 